\title{
Social Drone Sharing to Increase the UAV Patrolling Autonomy in Emergency Scenarios
}

\author{
Luca Morando*, Carmine Tommaso Recchiuto ${ }^{\dagger}$ and Antonio Sgorbissa ${ }^{\ddagger}$ \\ Laboratorium - Dibris, University of Genoa \\ Genova, Italy \\ Email: *luca.morando@edu.unige.it, ${ }^{\dagger}$ carmine.recchiuto@dibris.unige.it, ${ }^{\dagger}$ antonio.sgorbissa@unige.it
}

\begin{abstract}
Unmanned Aerial Vehicles (UAVs) popularity is increased in recent years, and the domain of application of this new technology is continuously expanding. However, although UAVs may be extremely useful in monitoring contexts, the operational aspects of drone patrolling services have not yet been extensively studied. Specifically, patrolling and inspecting with UAVs different targets distributed over a large area is still an open problem, due to battery constraints and other practical limitations. In this work, we propose a deterministic algorithm for patrolling large areas in a pre- or post-critical event scenario. The autonomy range of UAVs is extended with the concept of Social Drone Sharing: citizens may offer their availability to take care of the UAV if it lands in their private area, being thus strictly involved in the monitoring process. The proposed approach aims at finding optimal routes in this context, minimizing the patrolling time and respecting the battery constraints. Simulation experiments have been conducted, giving some insights about the performance of the proposed method.

Index Terms-Autonomous UAV, Optimization, Social Robotics.
\end{abstract}

\section{INTRODUCTION}

Multirotor drone technology is available on a large scale on market and widely utilized in research. Indeed multirotors are endowed with several specific features such as vertical take-off and landing, the ability to hover freely in the air, the reduced size and the possibility to be programmed, that make them very interesting in different scenarios. Thanks to all these characteristics, drones are now used in mapping [1], in agriculture [2], in inspection sites [3] and soon they may be also used in delivery. Emergency scenarios are another domain where UAVs are becoming increasingly important [4]. Indeed there are various examples in literature, starting from the nuclear disaster of Fukushima [5] to the pandemic disease caused from the virus Sars-Cov-2, where UAVs have been successfully employed: in particular, drones have been utilized to control the body temperature of a multitude of people in the street during quarantine periods [6]. Despite their effectiveness, a significant limit to the use of this technology lies in the absence of true autonomy. This problem is twofold:

- autonomous navigation behavior, i.e. the capability to autonomously coordinate a team of UAVs to perform a patrolling operations, is missing, thus requiring expert pilots also in the simpler operations.

- energetic autonomy, i.e., the capability to cover long distances without the need to be recharged, is limited to a time of flight of $15-30$ minutes, thus resulting in a reduced operative area.

Our proposal lies in the context of pre-operative or postoperative intervention in emergency scenarios, where rescue teams or the civil protection need to periodically monitor or survey different critical targets located in various places taking as little time as possible. These targets could be, for instance, fields or woods whose dryness shall be periodically assessed to prevent fires, soil slopes whose stability shall be monitored before or after floods, critical infrastructure (hospitals, schools, roads, bridges, etc.) whose risk shall be evaluated before or after earthquakes. In most cases the maximum drone flight time, generally defined by the drone technical specification, is not sufficient to visits all targets. For these reasons, the proposed approach aims to involve citizens in the monitoring process, by introducing the new concepts of Social Charging Stations (CS) and Social Drone Sharing (SDS).

More in detail we foresee a software architecture that is compose by different elements. A platform that permits to make in communication the authorities with the civil volunteers, making also them able to verify the status of the drones involved in the patrolling activity and their flight plans in real-time. Finally, by a computational architecture based on a multiple deterministic approach that permits to first maximize the coverage of the operational area given the availability of the resident volunteers and second to evaluate the optimal path for the UAVs taking into account the energy and the inspection constraints

Civil authorities can interface with the platform to change the targets to survey depending on the need, and also visualize the CS available in the operative area. Resident volunteers give their availability through a mobile application, which alerts the user if their help is required to prepare the landing spot and to recharge the batteries if the drone lands on their property. The purpose is to actively involved residents volunteers in risk assessment and management areas, which may be important especially in areas with low population density where a community-based approach is repaid by a greater safety.

Since resident volunteers are involved, which raises a number of logistics and legal issues, it is important to minimize the number of the charging stations required and, at the same time, maximize the inspection time in each target area. To this aim, this work describes an approach to find optimal routes 


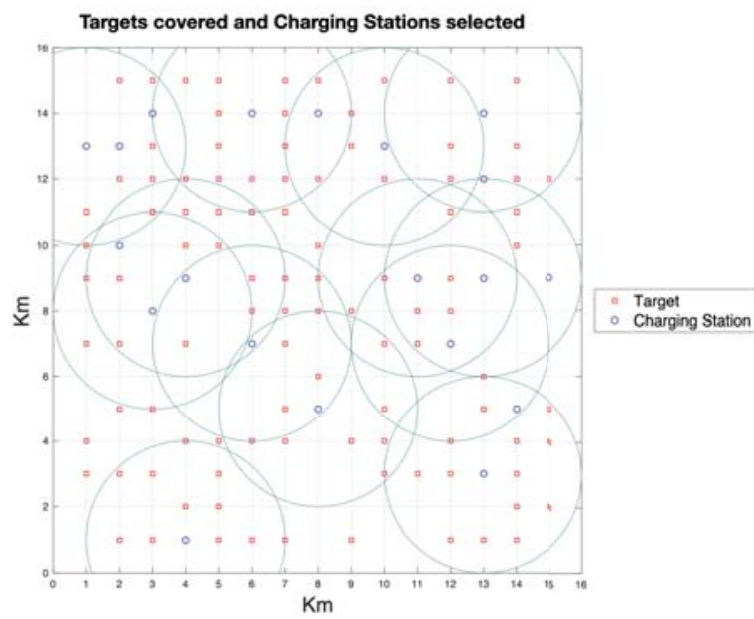

Fig. 1. Maximization of the target covering area via an optimal selection of the charging station place.

for autonomously flying UAVs in patrolling contexts, taking into account the new concepts of Social Drone Sharing.

\section{Problem Statement}

Our approach is based on the utilization of a single UAV, or a team of completely autonomous drones which are given by authorities to resident volunteers. The idea is that people give their availability in securing a recharging station spot in their property, such as a terrace or a garden, that a drone may use if it requires to be recharged. The availability of the resident volunteers are given as input to a first optimization algorithm, based on a MILP implementation, that permits to maximize the covering area, minimizing at the same time the number of CS involved. An example of this optimization tool is given in Figure 1, where the operative area is discretized and the possible locations for the targets to be inspected are located on the map. As visible in the figure, only the charging stations that permit to reach the maximum number of targets keeping the path continuity are chosen. In fact the criterion of selection is also based on the energy autonomy of the UAV utilized, indicated in the map via a circle (which ray length is based on the distance covered by the UAV with half of a charge) centered on each of the selected charging station. The solution consist in an optimal selection of the charging stations, maximizing the number of reachable targets keeping also the path continuity, as demonstrated by the overlap of different circles. The second optimization algorithm, expansively described in [7], take as input the targets and the charging station located in a better strategical position. The optimization procedure, based on a MILP algorithm defined by only two decision variables, draw the best path that the UAV have to accomplish in order to visit all the inspection targets, respecting also the energy constraints imposed by the batteries charge. An example of the algorithm is visible in Figure 2, where the performance are exploited in a realistic simulation located in a rural area,

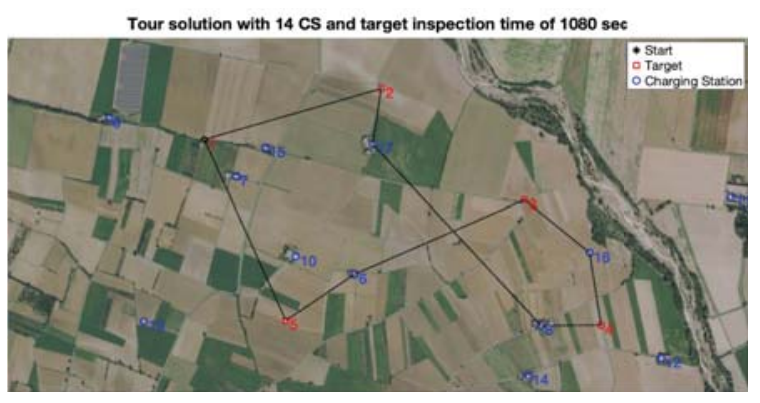

Fig 2. One solution of the iterative realistic simulation obtained with a number of 14 charging station and an inspection time $t_{i s p}=1080 \mathrm{~s}$ in each target.

where the continuous patrolling of the agricultural field is necessary. The charging stations are located near the house of the resident volunteers. In the evaluation of the optimal path, the energy autonomy (estimated in an empirical way) of a DJI Mavic 2 Pro is considered. Each target is associated to an inspection time required to complete the monitoring task. Figure 2 shows the final solution obtained after an iterative optimization process, where the inspection time required in each target is increased until no more solutions can be found. At this point more charging stations in various location are added to the map and the optimization process is repeated, obtaining the maximum inspection time in each target with the maximum number of available charging stations, finally placed on the map. In the solution depicted in Figure 2, a maximum inspection time of $t_{i n s}=1080 \mathrm{~s}$ is obtained with a total number of 14 charging stations and 5 targets. The computational time required by the optimization algorithm, running on Matlab on a Apple MacBook Pro equipped with an Intel Core i5 and $8 \mathrm{~Gb}$ of RAM, is about $t_{o p t}=5.9377 \mathrm{~s}$.

\section{CONCLUSION AND Future WORK}

This paper proposes a novel approach for the optimal routing of UAVs based on the new concepts of Social Charging Stations and Social Drone Sharing. Indeed, involving citizens in the monitoring process may be vital for increasing UAVs patrolling capabilities. The approach has been preliminarily validated with simulation experiments, analysing computational time and general performances, and eventually showing its feasibility.

Future extensions of this work concern the possibility of using more drones that share the Targets and their inspection time.

In any case, the possibilities to expand this work, to adapt it to a real patrolling scenario, are enormous, and all very interesting to explore, ultimately leading to a wider deployment of UAVs in critical contexts.

\section{REFERENCES}

[1] Patrik Schmuck and Margarita Chli, "Multi-UAV Collaborative Monocular SLAM," 2017 IEEE International Conference on Robotics and Automation (ICRA) Singapore, May 29 - June 3, 2017. 
[2] J. Gagoa, C. Doutheb, R.E. Coopmanc, P.P. Gallegoa, M. Ribas-Carbob, J. Flexasb, J. Escalonab, H. Medranob. (2015) "UAVs challenge to assess water stress for sustainable agriculture". Agricultural water management, 153, 9-19.

[3] Nadeem M Shajahan, Arjun Sasikumar, Thomas Kuruvila and Dhivin Davis, "Automated Inspection of Monopole Tower using Drones and Computer Vision" 2019 International Conference on Intelligent Autonomous Systems (ICoIAS).

[4] Recchiuto, C.T., Sgorbissa, A. Post-disaster assessment with unmanned aerial vehicles: A survey on practical implementations and research approaches (2018) Journal of Field Robotics, 35 (4), pp. 459-490.

[5] https://www.neimagazine.com/features/featurefukushima-looks-todrones-6728269/

[6] https://www.thelocal.it/20200410/hovering-police-drones-take-italianstemperature-and-issue-fines.

[7] Morando, L., Recchiuto, C.T., Sgorbissa, A.. "Social Drone Sharing to Increase the UAV Patrolling Autonomy inEmergency Scenarios", (2020), 29th IEEE International Conference in Human Robot Interaction (ROMAN), Naples, IT. 